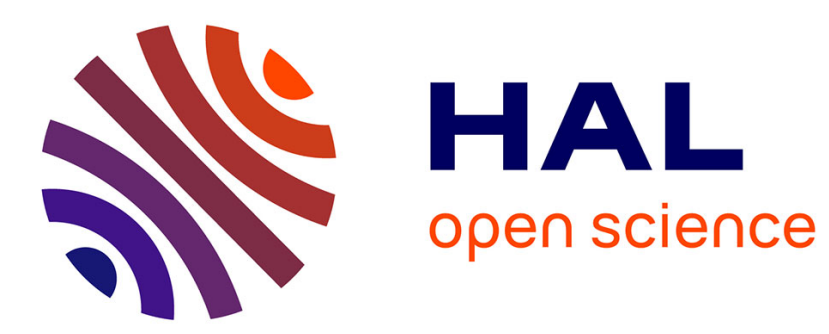

\title{
Twitter, machine à faire et défaire l'autorité
}

\author{
Louise Merzeau
}

\section{To cite this version:}

Louise Merzeau. Twitter, machine à faire et défaire l'autorité. Médium: Transmettre pour Innover, 2013, Récits du pouvoir. Pouvoirs du récit, 1 (34), pp.171-185. 10.3917/mediu.034.0171 . halshs00837547

\section{HAL Id: halshs-00837547 https://shs.hal.science/halshs-00837547}

Submitted on 22 Jun 2013

HAL is a multi-disciplinary open access archive for the deposit and dissemination of scientific research documents, whether they are published or not. The documents may come from teaching and research institutions in France or abroad, or from public or private research centers.
L'archive ouverte pluridisciplinaire HAL, est destinée au dépôt et à la diffusion de documents scientifiques de niveau recherche, publiés ou non, émanant des établissements d'enseignement et de recherche français ou étrangers, des laboratoires publics ou privés. 


\title{
Louise Merzeau,
}

\section{Twitter, ou la machine à faire et défaire l'autorité}

\author{
Médium n`34, 2013
}

Cet article est en partie repris, avec l'aimable autorisation des éditeurs, de «Twitter: une machine à fabriquer de l'autorité », in N. Pélissier, G. Gallezot, (dir.), Twitter, un monde en tout petit ?, Paris, L'Harmattan, collection "Communication et civilisation", 2013.

Lancée en 2006, la plateforme Twitter, qui vient d'atteindre 500 millions de membres, est sortie de la phase expérimentale où elle n'était utilisée que par des «primo-adoptants » et des professionnels de la communication. Non seulement l'outil de microblogging*1 est aujourd'hui pratiqué par toutes sortes d'utilisateurs, mais il joue désormais dans l'espace médiatique un rôle qui ne se limite plus à l'usage qu'en font les membres inscrits. Fréquemment relayés dans les mass media, les messages publiés sur Twitter sont soumis à de nouvelles modalités de propagation et d'interprétation qui compliquent les processus de filtrage et de légitimation des contenus.

Un nouveau système d'autorité se déploie, qui s'affronte et s'articule avec les anciens, dans l'ordre académique, médiatique ou politique.

\section{Publier ou fuiter?}

La clôture des échanges ne protège pas seulement l'intimité, mais aussi ce qui fonde l'autorité : la rétention d'information ou le secret. Parce qu'ils brouillent la frontière entre public et privé en multipliant les espaces intermédiaires ou hybrides, Internet et Twitter en particulier affectent donc aussi les rapports d'autorité attachés à cette distinction. De fait, il est aujourd'hui fréquent de voir s'affronter deux conceptions de l'autorité, l'une attachée aux cénacles, l'autre au réseau. Dans la première, c'est la confidentialité qui est un marqueur d'excellence et de pouvoir. Dans la seconde, c'est au contraire le fait de relayer l'information qui augmente son crédit auprès de sa communauté. S'il serait absurde d'ignorer tout ce qui s'est construit autour du principe de clôture, il le serait tout autant de réduire le microblogging à un dispositif d'exhibition inconvenante de ce qui devrait rester caché. Cette représentation repose sur une méconnaissance des mécanismes qui président à la propagation des messages, selon une distribution peut-être tout aussi complexe des degrés de légitimité, d'expertise et de notoriété. Le cas du live tweet* est ici symptomatique. Si l'on pense que la communication nuit à l'autorité et qu'elle n'est au mieux qu'une concession à faire de temps en temps au grand nombre (lecteurs, électeurs...), la pratique se résume à de l'indiscrétion. Mais on peut aussi considérer que la logique du microblogging n'est pas celle du dévoilement, mais au contraire d'une stratification supplémentaire - ou concurrentielle - des discours et des statuts. Si « tout le monde » peut en théorie accéder aux tweets, les messages ne sont en effet lus que par certaines catégories de lecteurs :

1 Les termes suivis d'un astérisque sont définis en fin d'article. 
- ceux qui les recherchent en formulant une requête ou en utilisant l'indexation par hashtag* dont on peut supposer qu'ils font déjà partie de cercles « bien informés »;

- les abonnés aux auteurs du live tweet;

- ceux qui suivent ces abonnés, dans le cas où les messages sont retweetés* ;

- les lecteurs de contenus extérieurs à Twitter, dans lesquels ces tweets peuvent être mentionnés.

Entre l'émission et la réception, s'interpose une série de filtres qui n'a plus rien à voir avec les mécanismes traditionnels de l'audience. Ici, la ségrégation des relais comme des messages est la condition même de la propagation, non le résultat d'une déperdition statistique de l'attention. C'est le paradoxe de l'effet réseau : la viralité vient du fait que la diffusion n'est pas radiale (d'un noyau émetteur vers des cercles de plus en plus larges), mais commutative (ouvert/fermé, pertinent/non pertinent) ${ }^{2}$ (Guillaume, 1998).

\section{Qui classe les informations?}

La figure traditionnelle de l'autorité, on le sait, suppose un surplomb qui s'incarne dans un ordre hiérarchique. Le numérique ne suspend pas cette nécessité de modeler l'information selon un certain relief, mais les processus qui produisent ces dénivelés diffèrent de ceux qui régissaient la graphosphère et la vidéosphère. Pendant un temps, le classement des contenus sur le Web a été monopolisé par Google. L'ancienne imagerie de l'autorité restait alors appropriée : suprématie, omniscience et opacité, entraînant adhésion (la pertinence des résultats n'est pas contestée par les internautes) et soumission (les règles de référencement imposées par le moteur sont adoptées par les webmasters). De fait, s'il restitue l'intelligence collective des internautes en valorisant les pages vers lesquelles pointent le plus de liens, le PageRank* est encore un système centralisé : il présuppose l'unicité d'un graphe des pages, comme celle de l'autorité méritocratique qu'il permet de mesurer. Comme l'a montré Cardon ici même ${ }^{3}$, d'autres systèmes de classement sont ensuite venus compliquer cette mise en ordre « autoritaire » du Web. Le comptage des « pages vues » et des « visiteurs uniques » a d'abord introduit dans le réseau des mesures d'audience héritées des médias traditionnels afin d'en importer les modèles économiques. Puis, le développement des réseaux sociaux a de nouveau bouleversé les métriques de l'autorité en les soumettant à la logique conversationnelle. Dans Facebook et Twitter, les classements sont affinitaires : la hiérarchie n’y est pas aplanie mais ramenée à la tension du j'aime/j'aime pas. On peut craindre qu'ainsi réduite au jeu binaire d'un presse-bouton, la qualification démocratique des contenus soit remplacée par une recommandation consumériste, téléguidée par quelques firmes toutes-puissantes. Mais ce que la dictature de l'affinité risque de contracter, la cooptation réticulaire contribue en même temps à le déplier. Car aucun réseau social ne saurait rétrécir : aussi sélectif soit-on dans le tri de ses « amis » ou de ses followers*, les cercles ont toujours tendance à s'élargir. Or avec la prolifération des connexions, se multiplient les filtres et les typologies. La fonction « liste » de Twitter en témoigne. Alors qu'elle vise à lutter contre la cacophonie du fil chronologique des tweets en isolant des groupes de personnes du bruit ambiant de la timeline*, elle sert aussi, en deuxième instance, à 
explorer les modes de classement des autres pour étoffer ses propres catégories et multiplier ses points d'entrée. Ainsi, les hiérarchisations sont alternativement fermantes et ouvrantes : chacun construit son petit système d'autorité, mais les autorités se branchent tôt ou tard les unes sur les autres pour former de vastes systèmes étoilés.

\section{Autoréférence, jeu et story-telling}

Aux internautes soucieux de leur e-réputation, les listes permettent aussi de vérifier dans quel groupe ils ont été eux-mêmes classés, par qui, et en compagnie de qui. Ainsi, c'est paradoxalement la possibilité théorique de participer chacun à la construction des taxonomies qui recréé continuellement de l'inégalité dans le réseau social. La dynamique contributive du microblogging entraîne en effet moins de la réciprocité qu'une réflexivité qui incite à continuellement hiérarchiser informations et locuteurs. Là où Facebook pousse à mettre tous les « amis » dans le même graphe, Twitter valorise au contraire l'écart entre followers et following, l'influence étant d'autant plus forte que le nombre d'abonnés et supérieur à celui des abonnements. Pour évaluer cet écart, chacun est incité à surveiller ses taux de "suivisme » et les mentions de son pseudo par le biais d'indicateurs intégrés à la plateforme ou d'API récupérant les statistiques des comptes pour les scénariser, les comparer ou les diffuser.

Comme dans un jeu, c'est de l'intérieur que l'utilisateur comprend progressivement ces règles et se risque à y prendre une part plus active. D'abord simple observateur, le novice apprend peu à peu à maîtriser l'impact de ses tweets (c'est-à-dire à choisir un style, un timing et un ton qui susciteront retweets et mentions) pour augmenter sa propre autorité. Là où la graphosphère indexait l'autorité sur la longueur du détour par un médiateur, la culture de réseau valorise ainsi une réflexivité couplant toujours davantage l'usage des outils à une activité d'auto-observation. Cette «médiation identitaire » ne signifie pas que l'individu pourrait s'affranchir de toute hiérarchisation, mais qu'il est au contraire de plus en plus contraint d'y prendre part.

Attestée par l'évolution des trending topics*, l'arrivée récente d'une nouvelle classe d'âge sur la plateforme accentue encore cette logique d'autoréférence et de jeu. Pour de nombreux adolescents, la pratique de Twitter est en effet synonyme d'une course à la popularité, ce qui suppose de développer de véritables compétences de community management* appliquées à soi. Faisant du gain de nouveaux followers une fin (certains en ont plusieurs dizaines de milliers), ils mettent en œuvre des techniques d'écriture et de partage destinées à augmenter leur notoriété : RT massif, follow back* et choix d'une ligne éditoriale autour du LoL* et du défi. Généralement liée à l'usage de pseudonymes (choisis avec soin), cette pratique ne revient pas à tout exhiber. Elle témoigne plutôt d'une intériorisation des mécanismes de traçabilité numérique, qui exigent à la fois de se rendre visible et de se protéger. La règle implique en tout cas d'inonder la plateforme de tweets (qui se comptent en centaines de milliers), parfois sous la forme d'une narration continue de soi. Microstory-telling, Twitter combine alors le modèle d'autorité de la célébrité télévisuelle à celui du personal branding*. 


\section{Confiance et proximité}

Chez les adultes, comme chez les jeunes, le fait de s'en remettre à ses following pour filtrer l'information indique une forme de soumission volontaire à l'influence d'un nombre restreint de gate-keepers, dotés d'une légitimité proche de celle que Weber qualifiait de «charismatique». Régulièrement exposés aux choix, aux avis et aux humeurs de ceux qu'il suit, l'utilisateur se place dans la position d'être conditionné par leur manière de dire et de penser. La difficulté d'exprimer des avis nuancés en 140 caractères, combinée aux effets d'une viralité qui se donne à voir en même temps qu'elle s'exerce, réduit d'autant l'aptitude des participants à garder une distance critique vis à vis de leur communauté.

Mais, plus qu'un système d'influence, le régime attentionnel de Twitter en fait surtout un vecteur d'autorité informationnelle, au sens où l'autorité y a pour effet d'in-former (donner une forme) ${ }^{4}$. Une pratique récurrente chez les utilisateurs les plus mûrs consiste à tweeter des liens vers des pages extérieures à la plateforme. Le microblogging contribue alors à redessiner en permanence les contours d'un Web actif, en générant du trafic, en affectant la cartographie des parcours, en orientant les commentaires. Les marques utilisent de plus en plus cette boussole attentionnelle pour augmenter la notoriété de leurs produits, et conséquemment leurs chiffres de vente. Mais, au-delà de la popularité, ce sont plus fondamentalement les distances informationnelles ${ }^{5}$ qui sont ainsi remodelées. La probabilité qu'un internaute se trouve confronté à une certaine information n'est en effet pas constante : elle dépend des couches médiatrices qui, en la rendant disponible, la rendent plus proche. Associant adressage et recommandation, les réseaux sociaux contribuent fortement à cette anamorphose ininterrompue, en diffusant des messages qui signalent, caractérisent et servent de point d'entrée vers les contenus en ligne.

\section{Des « petits mondes » à l'espace public}

L'augmentation du nombre d'utilisateurs n'a pas seulement démocratisé la pratique du microbloggig. Elle a aussi provoqué un glissement des «petits mondes »où se tenaient les conversations vers l'espace public : du régime de l' "entre nous », la plateforme a basculé dans le celui des médias de masse. Le relais, dans la presse et les JT, de messages envoyés par des internautes représentatifs ou célèbres modifie profondément la structure de leur propagation. Transposé dans le cadre énonciatif d'une rédaction, le tweet sort du système personnalisé des abonnements, des retweets et des mentions et rejoint la catégorie des petites phrases ressassées en boucle. D'une «machine à café 2.0 », où c'est le filtrage individuel qui fait la valeur des informations, on passe à un hall de gare numérique, où se télescopent des bribes de conversations coupées de leur contexte et de leur temporalité relationnels. Ce n'est pas le fait que les tweets soient publics qui entraîne une telle mutation (ils l'étaient déjà), mais l'ingérence d'une autre logique médiatique, où les contenus ne sont plus cautionnés selon les mêmes critères. Aux communautés

4 E. Broudoux, «Construction de l'autorité informationnelle sur le web », A Document (Re)turn : Contributions from a Research Field in Transition, 2007, http://archivesic.ccsd.cnrs.fr/sic_0012071

5 B. Rieder, «Pratiques informationnelles et analyse des traces numériques: de la représentation à l'intervention », Études de communication, n 35, 2010. 
restreintes (de pairs ou de proches), se substitue l'opinion publique. La parole n'est donc plus autorisée en priorité par la confiance, mais par la notoriété. À l'intérieur de la plateforme et de son écosystème d'applications, c'est la caution du groupe et le réseau serré de liens, de réponses et de renvois qui valide et enrichit les contenus tweetés. Dans la sphère médiatique, c'est le maintien d'un ordre consensuel garant d'une synchronisation du collectif qui sélectionne certains messages pour en accroître l'impact attentionnel.

\section{Autorité, sans œuvre ni auteur}

La blogosphère s'est précisément attaquée à cet ordre médiatique, en faisant émerger une nouvelle catégorie d'auteurs en dehors des circuits éditoriaux classiques, « en leur conférant une "autorité" à partir des actes épars de lecture et de transmission (citation, reprise, lien, etc.) » 6 . Twitter pourrait également relever de cette éditorialisation inversée, où l'édition repose sur une accréditation collective a posteriori. À bien des égards, le microblogging peut d'ailleurs être considéré comme une activité satellite alimentant le monde des blogs, renforçant la notoriété de certains blogueurs par les conversations que leurs billets suscitent.

Une telle vision risque cependant de réduire le Web à l'activité des plus productifs. Or si une partie des messages consiste bien en une autoédition, l'immense majorité des conversations se tient en dehors de toute production « originale ». L'écriture la plus courante relève moins d'une création que d'une souscription : "On ne navigue plus, on ne recherche plus, on s'abonne, on "souscrit". [...] "Souscrire", "sub-scribere", littéralement "écrire en dessous", à moins qu'il ne s'agisse d'écriture "sous autorité" ${ }^{7}$. Dans le flux incessant des messages qui défilent sur nos timelines, plus personne n'est véritablement un auteur. Curateurs de nos propres affinités, nous ne signons nos contenus qu'au deuxième degré, sur le mode de la citation, du vote et du relais. L'auteur collectif et décentré que plébiscite la blogosphère postule encore la cohésion d'une œuvre, quelque part entre l'amont et l'aval d'un flux pensé comme déroulement, postproduction et promotion. Le modèle s'applique donc mal à la fragmentation des contenus dans les réseaux sociaux, qui fabriquent de l'autorité sans œuvre. Dans Twitter, ce que les hiérarchies servent à autoriser n'est plus la chose publiée, mais le partage lui-même comme nouvelle plus value. Cette «vaporisation » de l'autorité s'inscrit dans ce que Doueihi a identifié comme l'une des tendances fondamentales de la conversion numérique : «le partage anthologique, [...] où [...] au lieu d'être nécessairement lié à des auteurs, avec leur identité ou leurs intentions, le sens l'est plutôt à une catégorisation flexible (ou à un "étiquetage" par mots-clés) ${ }^{8}$.

Si aucun contenu ne saurait plus se propager sans être personnalisé, la personnalisation ellemême s'exerce sur le mode de la dissémination. La circulation et la décontextualisation des traces

6 E. Broudoux, Ph. Bootz, et alii, « Auctorialité : production, réception et publication de documents numériques », La redocumentarisation du monde, 2005, http://archivesic.ccsd.cnrs.fr/sic 00120699

7 O. Ertzscheid, «Les 5 moments de l'écriture en réseau : les moteurs comme scripteurs », Affordance, 22/02/2011, affordance.typepad.com/mon weblog/2011/02/les-5-moments-ecriture-web-reseau.html

8

M. Doueihi, La Grande conversion numérique, Éditions du Seuil, 2008p. 70. 
numériques les rattache à une énonciation paradoxale où l'individu est (d)écrit autant qu'il est (d)écrivant :

- par les membres de la communauté qui interagissent avec lui,

- par la sphère médiatique qui se greffe sur ses conversations,

- par les machines enfin, qui répliquent, indexent et calculent la moindre de ses émissions.

Sur le réseau, l'autorité c'est d'abord une grandeur mesurable indexée sur une série de variables (nombre d'abonnés ou d'amis, taux de retweets et de mention, fréquence de publication, amplitude du graphe social, etc.). À mesure que les écritures de souscription se sont généralisées, les algoritmes ont en effet occupé la place laissée vacante par les auteurs. Cette autorité calculée se traduit en scores, que les plateformes et leur écosystème d'API se chargent de recenser, publier et exploiter. C'est le cas de Klout, qui mesure l'indice d'influence des internautes et les classe en broadcasters, taste makers, socializers, explorers, etc. Cette métrique n'intervient pas seulement après coup, comme une validation de la présence en ligne. Elle agit aussi en amont en étant exploitée par les moteurs de recherche. Google ou Bing incluent en effet de plus en plus dans leurs algorithmes un facteur social, en triant les contenus en fonction de ceux qui les recommandent.

Cette emprise de l'algorithmie peut aller jusqu'à redéfinir l'autorité comme un coefficient purement probabiliste. L'exploitation de plus en plus courante de Twitter à des fins de prédiction va dans ce sens. Ici, ce n'est plus la hiérarchie des scores d'influence compte, mais la fréquence des messages traitant d'un même sujet, dans la mesure où elle indique une tendance qui permet d'anticiper (les cours de la bourse, un mouvement social, le palmarès des Oscars ou l'apparition d'une épidémie ${ }^{9}$. Ce qui fait alors autorité, c'est la sagesse statistique des foules, telle qu'elle est enregistrée par les capteurs des applications.

\section{«Nous ne faisons que nous entregloser » ${ }^{10}$}

Dans le contexte qu'on a décrit, que reste-t-il de l'auctorialité si ce n'est un art de re-tweeter, recopier, re-cycler? Dans le temps long, le «travail de la citation » a toujours été l'envers des formes successives d'institution de la parole autorisée ${ }^{11}$. La fragmentation, l'étoilement et le nivellement des instances auctoriales commencent avec la contamination de l'espace livresque par la dispositio du journal, dont Mallarmé avait pressenti le pouvoir destructeur autant que structurant. Après la « cacographie » sur laquelle se ferme la graphosphère, l'hypersphère ajoute ses propres chapitres : l'âge "autoritatif » des blogs, où les auteurs construisent eux-mêmes les conditions de leur reconnaissance. Puis le tout-citation de Twitter, où tous les messages sont des coups de dés qui se joueraient dans des constellations algorithmiques. Cherchera-t-on à reprendre la main sur les mécanismes de l'autorité ou les abandonnera-t-on toujours plus à l'intelligence calculatoire des données ? Difficile de répondre, tant il est vrai que les tendances lourdes (écritures industrielles et traçabilité) peuvent toujours être courbées par les contrecoups du détournement et de la réappropriation.

9 Fr.-B. Huyghe, « Prédiction $2.0 »$, huyghe.fr, 2/11/2011, http://www.huyghe.fr/actu 986.htm

10 Montaigne, Essais, III, 13.

11 Voir A. Compagnon, La Seconde main, ou le travail de la citation, Éditions du Seuil, 1979, 408 p. 


\section{Lexique}

PageRank: algorithme de sélection du moteur de recherche de Google privilégiant la notoriété des pages publiées sur le web.

Microblogging : mode de publication sur le web apparenté au blog (blogging) caractérisé par la brièveté des messages postés (140 caractères dans le cas de Twitter).

Live tweet (LT) : suivi en direct d'une réunion ou manifestation sous forme de messages publiés sur Twitter par des personnes assistant ou participant à l'événement, permettant de le suivre à distance et de dialoguer avec les participants.

Hashtag : mot clé permettant d'indexer librement les tweets et de constituer par ce bais des groupes de discussion, tout en facilitant les recherches thématiques dans le « corpus » de Twitter.

Retweet, retweeter : retransmission d'un tweet (fonction intégrée à la plateforme)

Twittos : utilisateurs de Twitter.

Follower, following : les followers sont ceux qui «suivent» un compte Twitter et les following ceux qui sont suivis. La notoriété d'un twitto est déterminée par le nombre de ses followers.

Followback: échange de bons procédés entre followers et followings : « je te suis si tu me suis ».

Community management: gestion ou modération de la «communauté » des internautes qui interviennent sur une page ou un site.

Timeline (TL) : fil chronologique des tweets que reçoit un utilisateur de Twitter en fonction de ses abonnements (les plus récents remplaçant les plus anciens).

Trending topics (TT) : calcul statistique des mots, hashtags ou noms propres les plus tweetés, déterminant des thèmes ou préoccupations tendance.

Personal branding : gestion de son identité numérique sur le modèle de la gestion de marque.

LoL (Laughing out loud) : interjection en forme d'acronyme pour désigner une publication présumée drôle, ou pour signaler que cette publication est « pour rire ».

Louise MERZEAU est maître de conférences HDR en sciences de l'information et de la communication à l'université Paris Ouest Nanterre La Défense et directrice du laboratoire Tactic (Traitement et appropriation des connaissances par les TIC). Elle a notamment coordonné un numéro de la revue Hermès sur la traçabilité sur les réseaux et publié de nombreux articles sur la présence numérique.

www.merzeau.net 